\title{
Explicit representations of common artefacts in simulation
}

\author{
Dr. Jon W. Hand ${ }^{1}$ \\ ${ }^{1}$ Energy Systems Research Unit, University of Strathclyde, Glasgow, UK
}

\begin{abstract}
Buildings are populated with artefacts which exhibit complex response characteristics to the surrounding environment. Computers, copy machines, ovens and electric storage heaters have sometimes-substantial temporal shifts between their inputs and the casual gains they introduce into rooms. Are these thermophysical complexities simply noise that can be ignored or can they have a bigger part to play in our virtual environments?

Abstract representations can make it difficult for whole building numerical tools to match experimental measurements or short term detailed observations. There are many possible approaches to reducing descriptive and thermophysical abstraction. This paper explores the implications of treating sub-room scale artefacts as one or more explicit thermal zones and use the solution techniques applied to thermal zones to capture these elusive response characteristics. The paper also explores the influence of sub-room scale artefacts on the rooms and occupants. The paper also explores whether this makes a noticeable difference to the patterns of performance recorded within the built environment and the computing resource implications of such an approach.
\end{abstract}

\section{Introduction}

If we choose to include in our virtual worlds representations of furniture and fittings within buildings we have access to their thermophysical interactions with the building fabric, occupants and environmental controls. The current study is done in the context of ESP-r via the dynamic, multi-domain solution techniques described by Clarke (2001) as well as the Radiance visualisation suite Larson (1997).

In Figure 1 everything visible is also thermally explicit the chairs and desks are real and there is paper in the filing cabinets as well as zones for ducts and air handlers and all are treated with the same level of virtual physics as the facade. Portions of this office also include CFD domains, but this is not reported in this paper. For this study sufficient information was not available to support 2D or 3D conduction so $1 \mathrm{D}$ conduction is used.

This increase in the resolution of the model and allows us to explore local comfort issues and better represent the temporal response to changes in boundary conditions, environmental controls and room use.

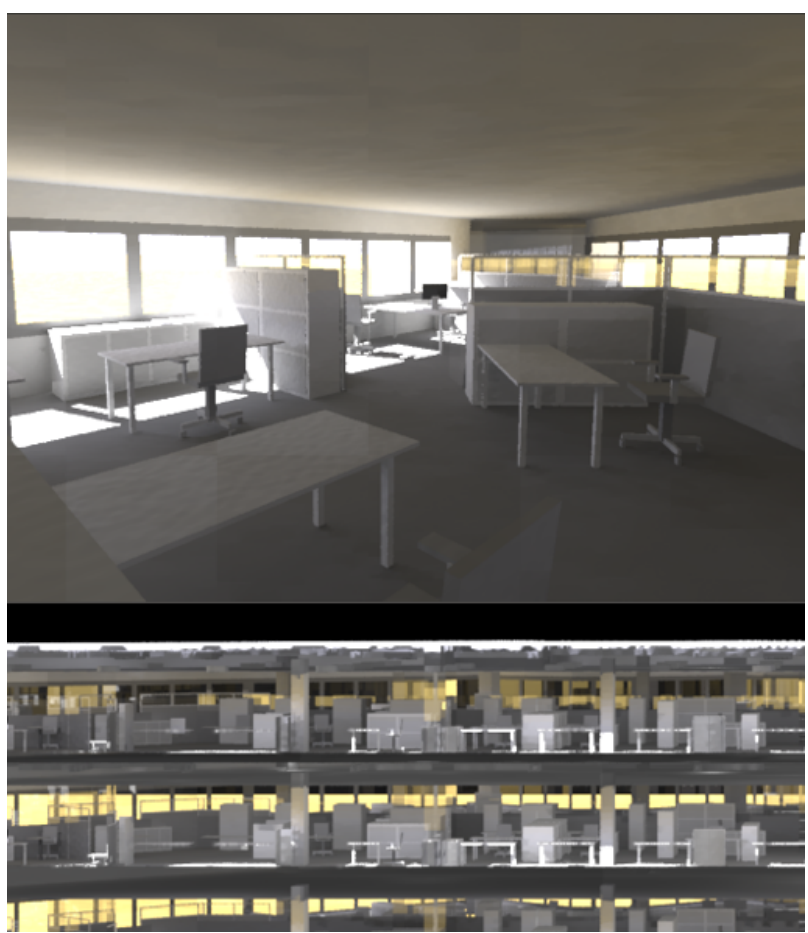

Figure 1: explicit thermal and visual model of office.

This paper extends the analogy to explore what happens if we explicitly represent:

- the form and composition of a thermostat case, circuit board and sensor - each fully represented in the energy balance and solved dynamically along with its surrounding environment.

- the form and composition and temporal heat generation and distribution of ubiquitous equipment in offices such as photocopy machines.

- components of a wet central heating system and their dynamic interactions with the rooms they are in and their impact on local comfort.

- a mechanical ventilation with heat recovery (MVHR) device and compare it with a system component based approach.

\section{Explicit sensors}

Various approaches have been used to describe sensors used by environmental controls in building simulation tools. The what is being sensed and the location of the sensor has considerable variety. In ESP-r both ideal and component based environmental controls supports: 
- sensing zone dry bulb temperature with a radiant and convective split

- sensing the temperature at a surface or a node within a surface

- sensing a weather parameter

- ideal controls can have weighted responses to sensors in multiple locations

In EnergyPlus (2017) there are a number of key words such as:

- ZoneControl:Thermostat/ZoneControl:Humidistat

- ZoneControl:Thermostat:OperativeTemperature

- ThermostatSetpoint:ThermalComfort:Fanger

Such key words and their attributes specify what is sensed within the zone (but not a position in the zone). There is also a displacement stratification model allows the user to specify a height and a calculated temperature is returned as well as options for sensors related to hydronic systems. Thermostats approximating users are based on the selected thermal comfort model for any given time period.

And what of the abstractions in the simulation entities that generate these control signals? Reality check: thermostats tend not to sense dry bulb temperatures (certainly not those attached to wet central heating radiators) and they certainly do not respond immediately.

According to Cockroft (2018), we must look at the inner construction of thermostats to see if the sensor is mounted on the circuit board (as in Figure 2), raised above the circuit board or attached to the thermostat case. The sensed condition then become the circuit board or a mix of the air and case temperature or the thermostat case itself. Modern thermostats tend to be poorly aspirated and some manufacturers design in a slow response to limit system cycling.

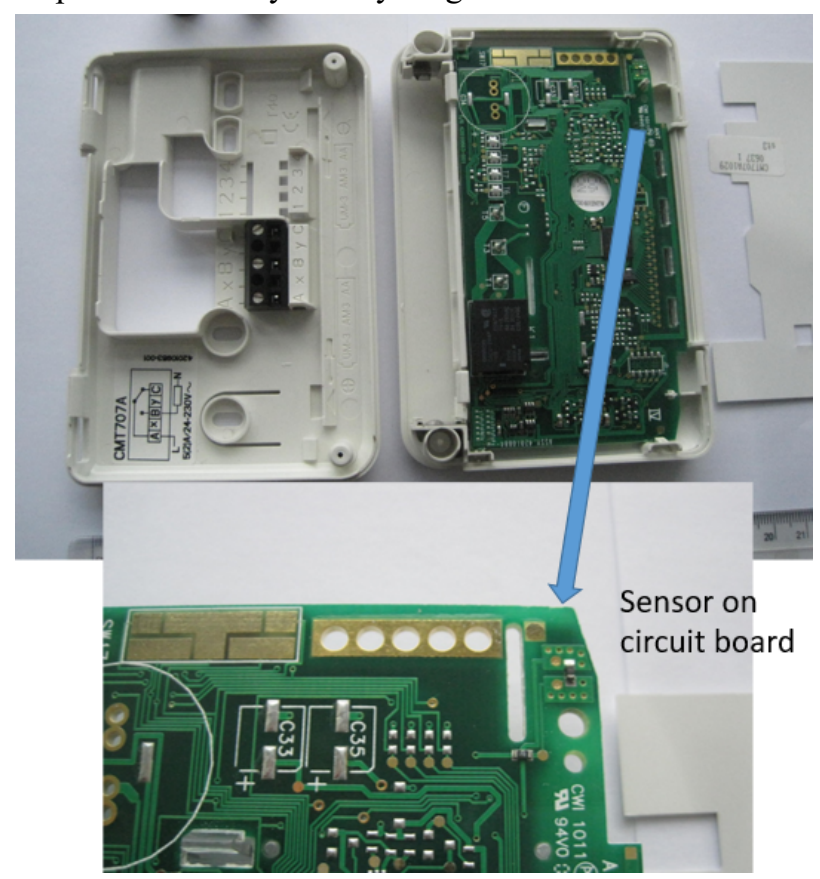

Figure 2: Thermostat layout.
A classic disconnect between abstract assumptions and observed responses is where a thermostat is mounted in a position that occasionally is in a sun-patch. To demonstrate, an explicit Honeywell unit is included on the side-wall of the lounge of a residential model which will be used in several of the case studies (see Figure 3).

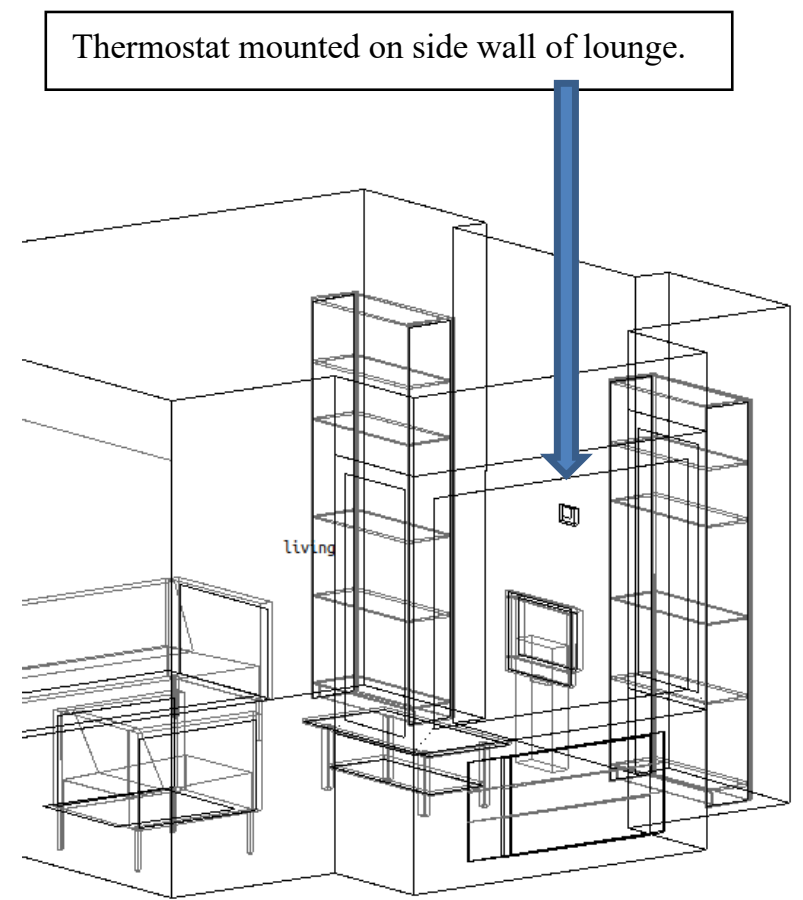

Figure 3: Context of explicit thermostat.

Here the sensor is soldered to the main circuit board (see Figure 2) and the zonal representation in Figure 4 includes the case, the circuit board as well as the battery and sensor. There is limited aspiration via crack components in the mass flow network (CFD at this scale of domain has not been validated so was not attempted). The sensor participates in the living zone hourly insolation calculations and the radiation view factors between the case and the surfaces in the room have been calculated.

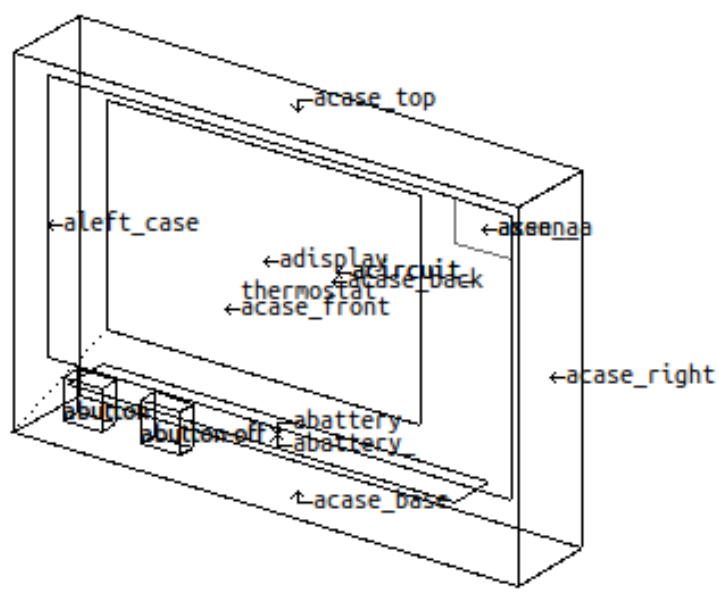

Figure 4: Zonal representation of thermostat. 
The hourly basis for ESP-r's computation of insolation patterns in the room somewhat obscures the timeresponse of the thermostat. The literature provides few clues about the rate of air flow within thermostats so that could also impact the accuracy of the approach.

The difference between the reported zone dry bulb temperature, zone resultant temperature and the thermostat case and sensor temperature are shown in Figure 5 where the sun patch is and issue from $\sim 14 \mathrm{~h} 00$ 15h30. Note also that the thermostat tends to lag the reported dry bulb temperature.

Lib: 3BR det obi3 win1 18Konoff.res Set: 2: For 3BR det obi3 with TRV control
Period: Thu-08-Feb@00h03(2007) to Thu-08-Feb@19h59(2007) : sim@01m, output@01

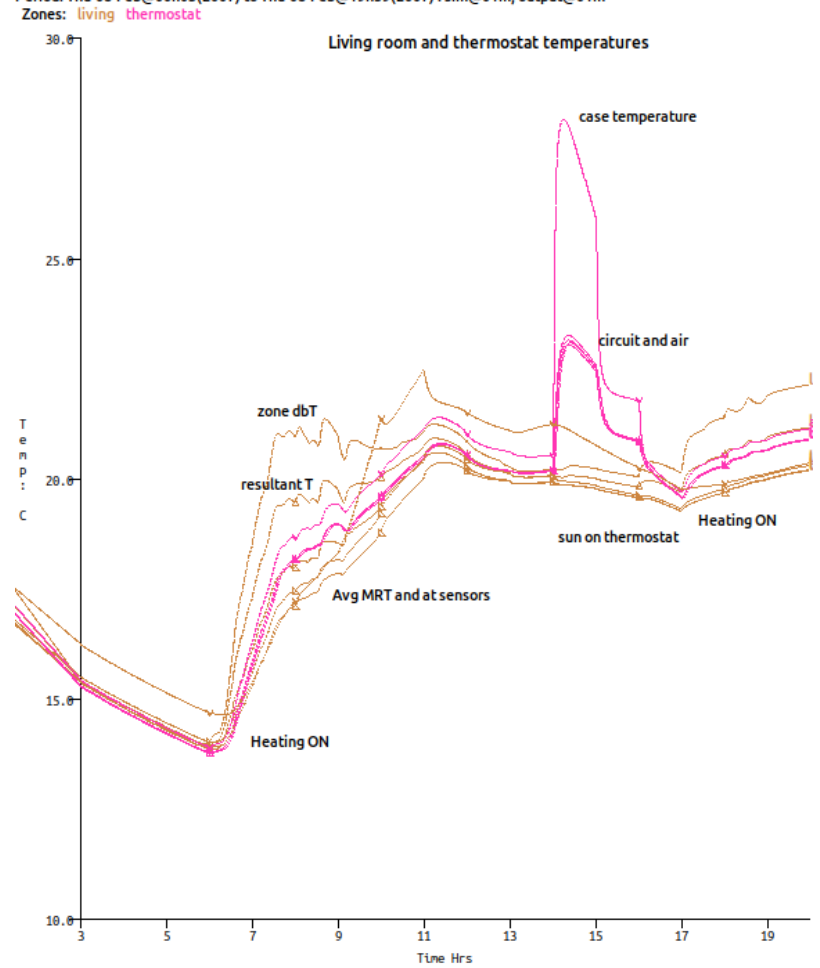

Figure 5: Different sensed conditions

\section{Explicit system components}

Environmental systems in simulation tools tend to respond immediately to control signals. Figure 6 shows heat injections from an ideal heat injection controller in ESP-r. Other tools also typically show sharp transitions which are unlikely to match observations.

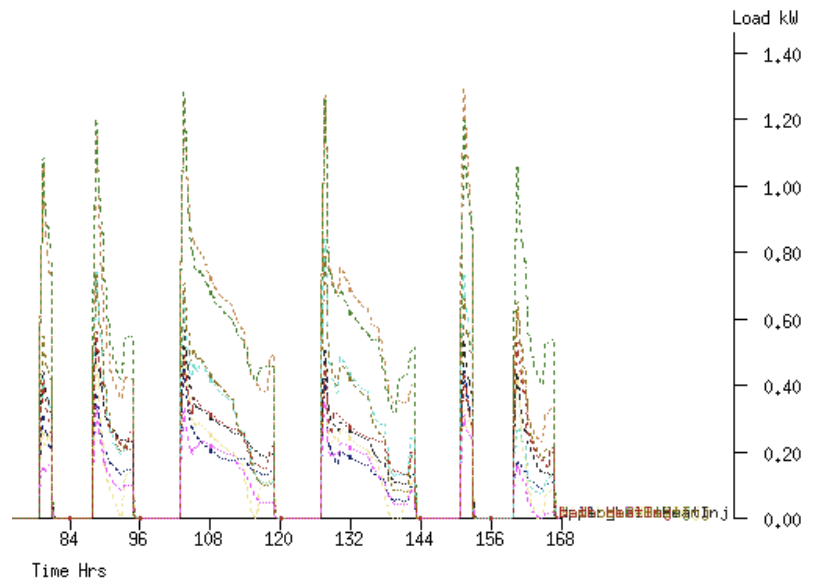

Figure 6: Instant response to control signals.
However, step changes in injection of heating and cooling flux does not fit with observations of many real systems. It may take 20 minutes for a domestic boiler to reach its operating temperature and heating persists for similar periods after pumps stop running. Air based systems are not immune - the thermal mass of ducting time-shifts delivery temperatures. The graph in Figure 7 shows measurements at a domestic boiler and two radiators during a morning start-up period. After 20 minutes the boiler firing cycles and at about 26 minutes the boiler shuts down and the temperatures gradually return to ambient temperature over the next hour.

A thermographic study of the temperature regime within the kitchen and lounge radiators from Figure 7 as shown in Figure 8. The upper is the kitchen $\left(\Delta \mathrm{T} \sim 10^{\circ} \mathrm{C}\right)$ and the lower is for a long-low radiator in the lounge $(\Delta \mathrm{T}$ $\sim 15^{\circ} \mathrm{C}$ ). Stratification which becomes less extreme as the room approaches the desired temperature and persists during the cool-down cycle where $\Delta \mathrm{T}$ approaches $3^{\circ} \mathrm{C}$.

Observed WCH temperatures (minutes from pump-

on

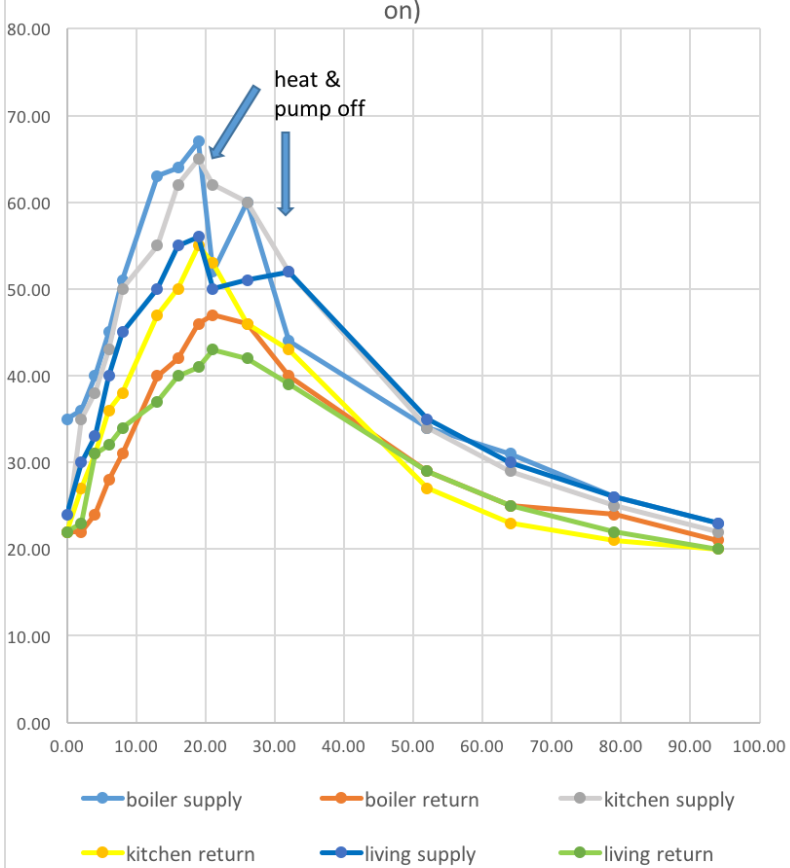

Figure 7: Measured temperatures in WCH system.

Earlier papers (2016) discussed high resolution residential models which included explicit furniture and fittings. These models used water filled thermal zones for radiators, the water jacket of the boiler and a water mass flow network to distribute the fluid (Figure 8). The model tracks the dynamic convection and radiation exchanges as well as the thermal inertia of the fluid mass and casing and their comfort impact on nearby occupants. Although a CFD domain was used to represent the combustion chamber in the earlier study, this was not included in the current study so that longer assessment periods could be studied.

However, a single temperature for the fluid in a radiator clearly does not reflect observed temperature distributions seen in Figure 9. For this study a higher 
resolution variant has been created, where each radiator is composed from three horizontal water filled and steel bounded zones which are linked via the flow network and with individual controls for the thermostatic radiator valves sensing operative temperature (two styles of radiator are shown in Figure 10).

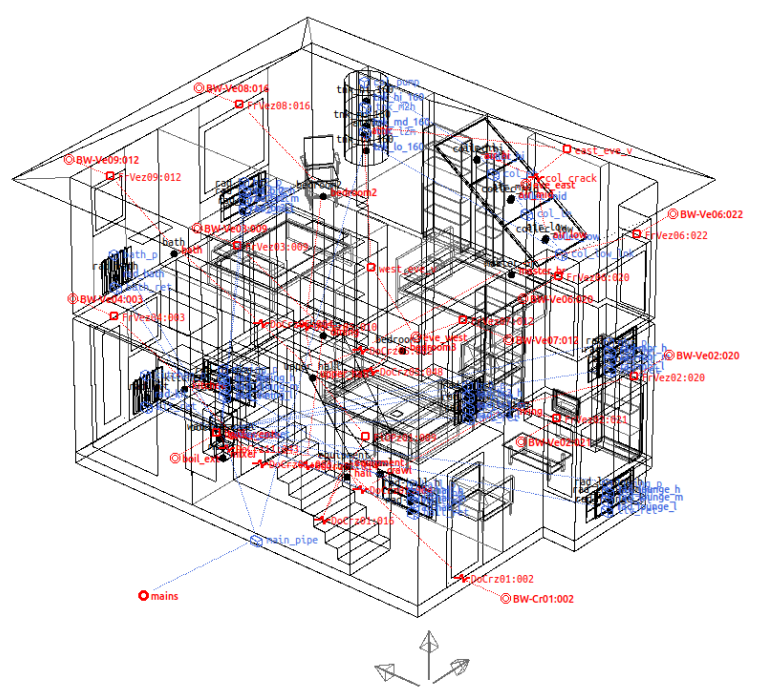

Figure 8: High resolution residential model.
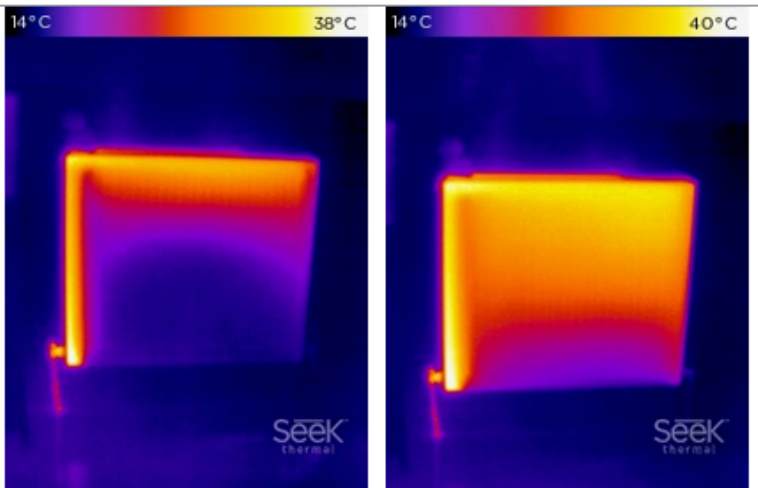

Initial flow

stable flow
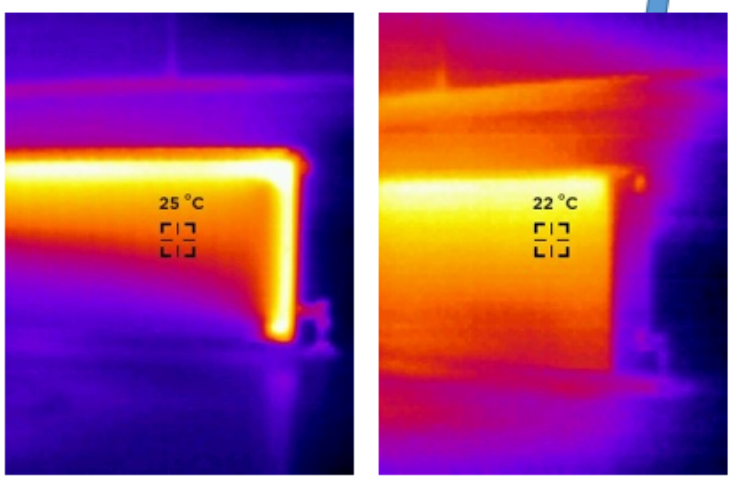

Initial \& cool-down stratification in long-low radiator

Figure 9: Thermographic study of stratification.

Calibration of the model proved to be an iterative process. An initial idealized control study determined the rough capacity needed in each room (Figure 6). Predefined radiator entities were imported into the model and the flow rates were adjusted to reflect the capacity of the boiler. The initial overall ideal capacity of $10 \mathrm{~kW}$ resulted in an extended start-up period. After several iterations a $16 \mathrm{~kW}$ capacity resulted in warm-up periods which approximate observations. It was also found that a two minute time step provided the necessary resolution for control of the system and to ensure that the injected heat was absorbed correctly in the boiler water jacket.

The model was simulated for various times of the year and one day in February captured for presentation. In Figure 11 gas firing is the upper grey line and the water jacket takes about 30 minutes to reach is normal operating temperature. There is stratification within the three radiator zones and cycling of the thermostatic radiator valve can be seen as the room reaches set-point. The extended cool-down is also evident. The dry bulb temperature, resultant temperatures and MRT at occupants follow a similar pattern to that in Figure 7.

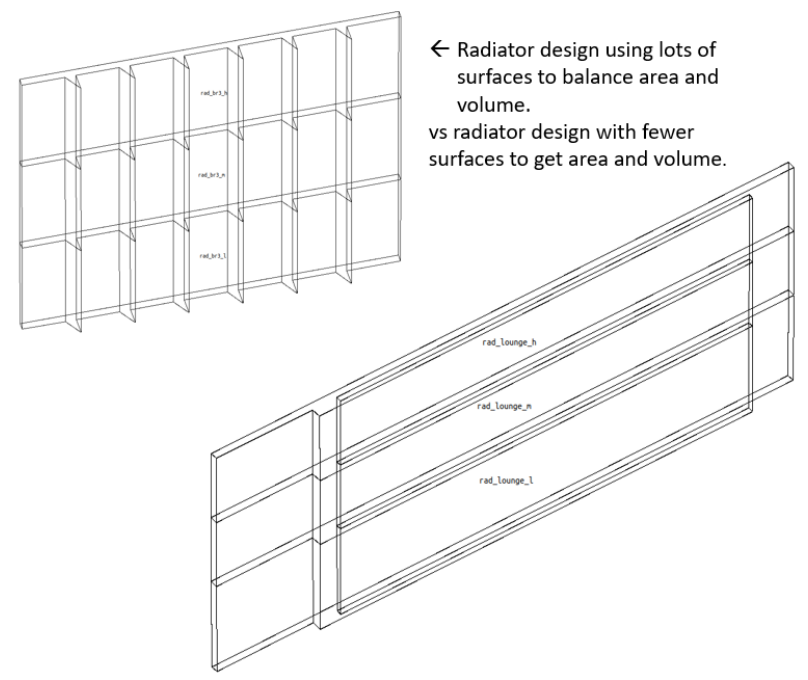

Figure 10: Wet system flow components and zones.

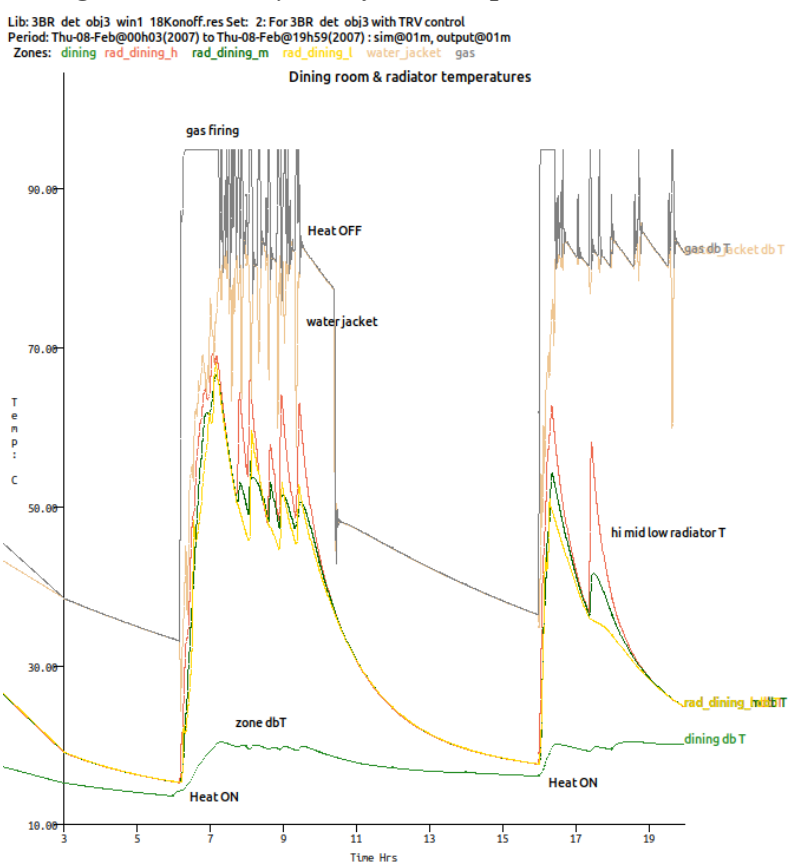

Figure 11: simulated WCH system components. 


\section{Office equipment}

Photocopy machines are ubiquitous. As sources of heat in rooms, their operation involves complex temporal patterns of warm air and warm-to-hot surfaces which are rarely captured within whole-building simulation tools. What are the implications of using abstract representations and typical hourly values of heat injection vs more explicit representations which might better track observations?

The printer room shown in Figure 12 includes a large format plotter, a small radiator which is never used, as well as a mid-range A4-A3 colour photocopy machine with paper sorter. The room includes a small extract grill similar to that in adjacent cellular offices to maintain fresh air levels. The door is always left open to the adjacent passage so elevated temperatures tend to disperse via mixing. On two sides are conditioned cellular offices and two sides are passages. Three of the walls were lightweight and one concrete block. There is a ceiling void above the room. Lighting tended to be off in the room unless detailed work was underway.
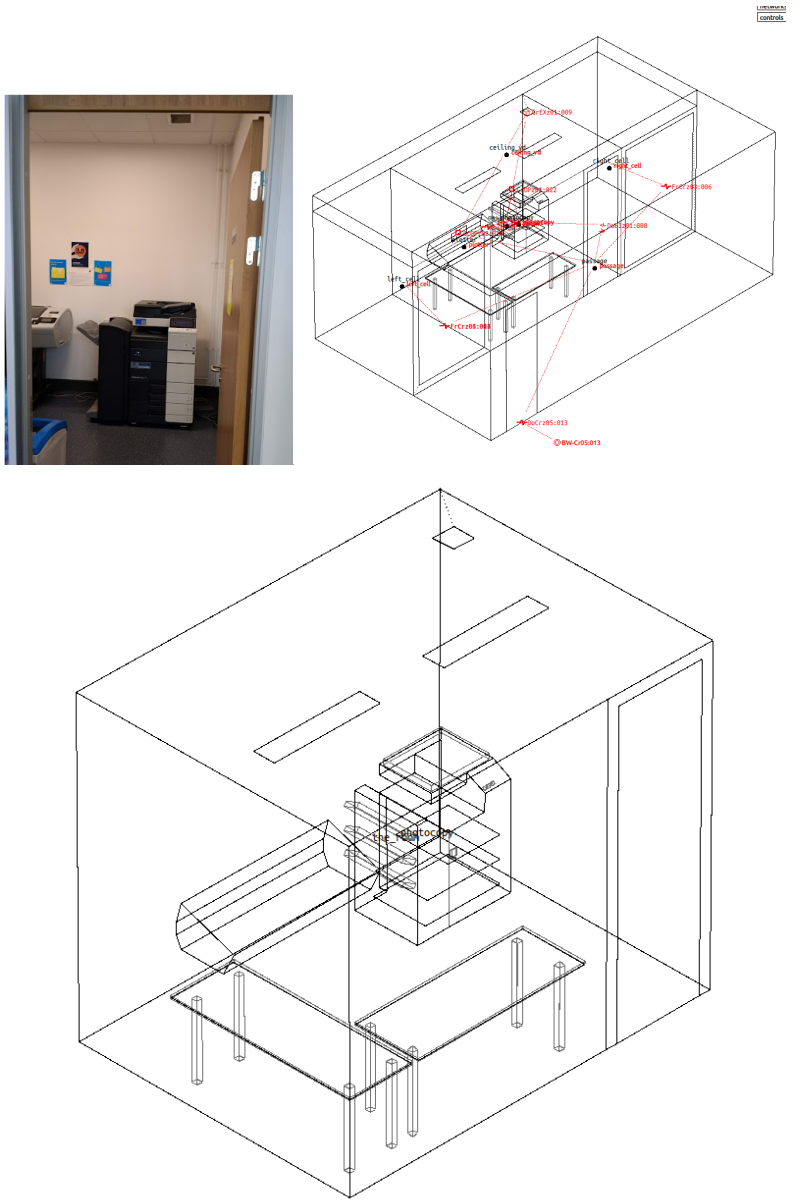

Figure 12: Photocopy room and model.

A thermal scan of the room and photocopy machine (Figure 13) indicated the case temperature varied between $24-38^{\circ} \mathrm{C}$. Vents in the device introduced air into the room in the $30-45^{\circ} \mathrm{C}$ range and newly printed paper emerging at $\sim 55^{\circ} \mathrm{C}$. Air near where users interacted tended to be $30-34^{\circ} \mathrm{C}$. The machine standby state seemed to be rarely, if ever engaged.

To characterise the temporal response, temperatures near the printer as well as within the room were measured minutely. A clamp meter tracked the power demands of the copy machine at 10 s intervals with a back-up Watthour meter. Standby mode (10-100W) was observed on weekends as well as 1-2 hours after staff had departed. First use varied from $8 \mathrm{~h} 30$ to $10 \mathrm{~h} 00$. The warm-up sequences would draw $\sim 1100 \mathrm{~W}$. Print runs of several hundred pages would draw $\sim 800-900 \mathrm{~W}$. Shorter runs $\sim 500-600 \mathrm{~W}$ dropping to $\sim 100 \mathrm{~W}$ for the next 30 minutes or the next use. The keep-warm function tended to draw $100-300 \mathrm{~W}$ (with rapid changes of state). Because this device was often lightly used this tended to be the dominate state during office hours. Scanning facilities made fewer demands and where not specifically tracked.
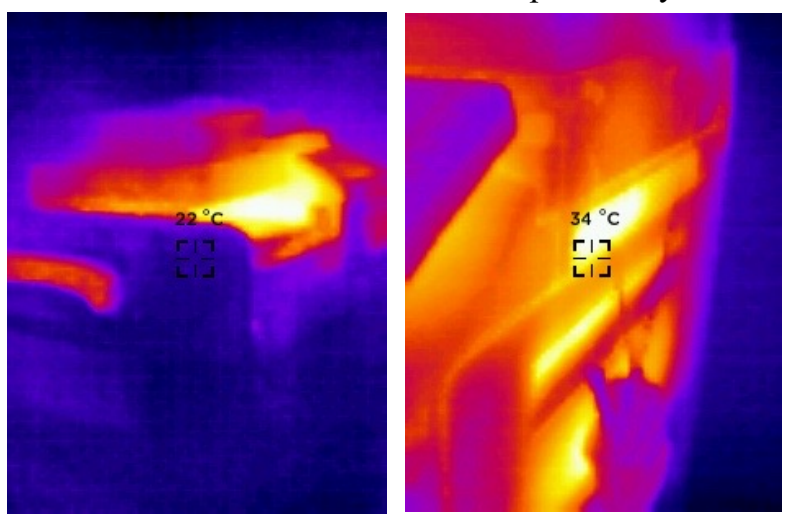

Figure 13: Heat emissions from photocopier.

Lib: photocopy room adjishc fanb.res: For photocopy room adj fans \& hc Period: Mon-08-Jan@00h00(2007) to Tue-09-Jan@23h59(2007) : sim@01m, output@01m Zones: photocopy

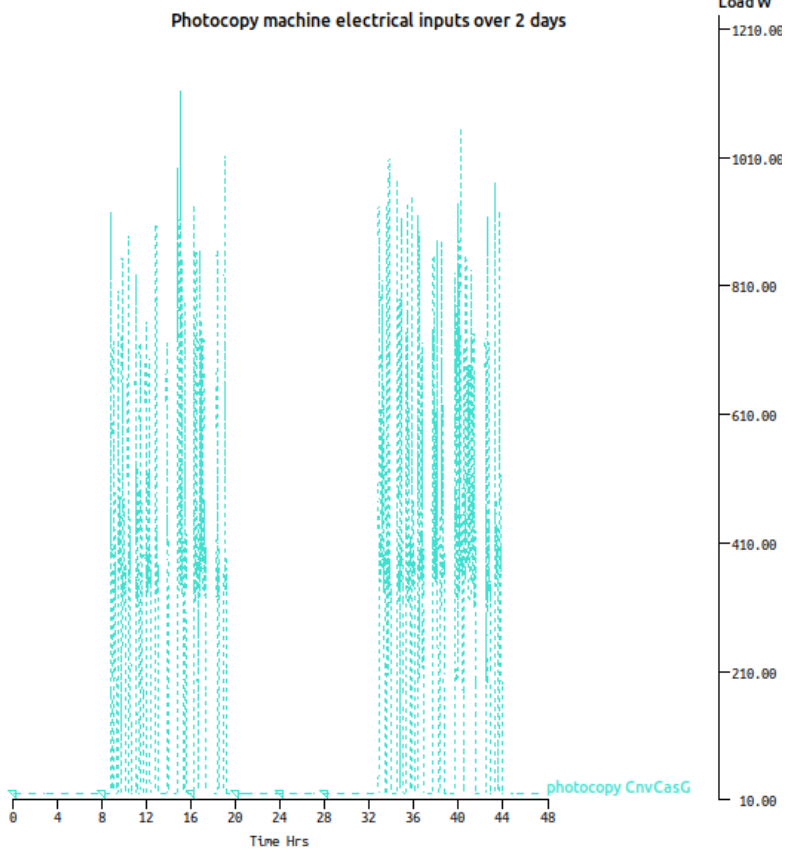

Figure 14: Measured electrical use imposed on the zone.

Temperatures near the paper exits and under the scanner were typically $30-34^{\circ} \mathrm{C}$ whether or not the device was actively printing. During office hours the room 
temperature would gradually rise and stabilise $3-4^{\circ} \mathrm{C}$ warmer than adjacent spaces.

An explicit zonal model of the device was created which which has the same surface area and roughly the same composition. The internal components and mass were represented with a number of steel plates and these were also used for portions of the case where elevated surface temperatures were observed. After testing this was encapsulated as a pre-defined entity (a mix of ESP-r surfaces and Radiance entities) which was imported into the ESP-r model. The bounding surfaces of the device then were included in the room zone and explicit radiation view-factors were calculated between all of the surfaces. Measured minutely electrical demands (Figure 14) were used as casual gains within the zone and its cooling fans and air circulation represented via controlled (on if the device core exceeded $40^{\circ} \mathrm{C}$ ) air flow components.

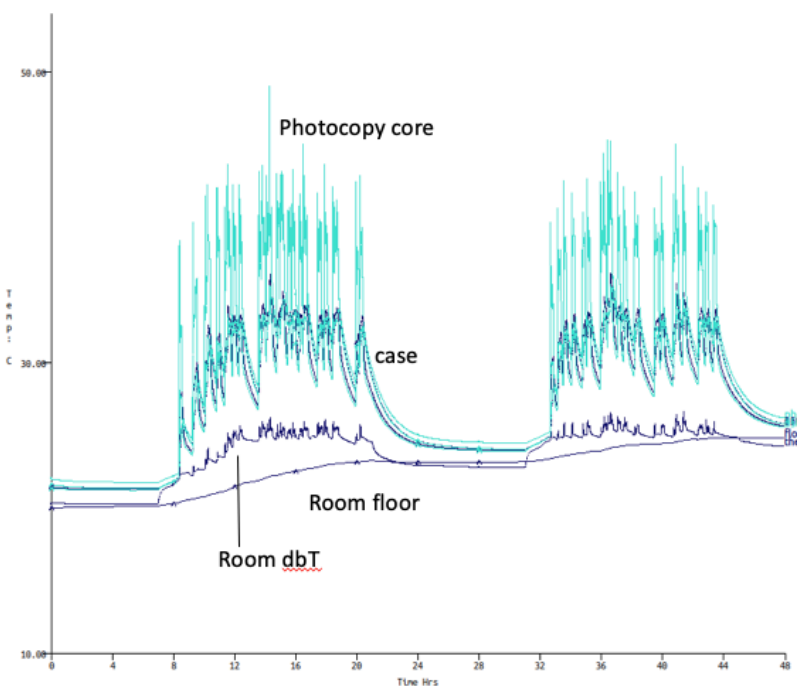

Figure 15: Predicted room \& photocopy temperatures.

Uncertainties in the model were:

- flow rate for the device fans and non-forced vents

- variation in temperature and heat generation within different sections of the device

- the length of time which newly printed paper was left in the sorter

- case material composition

- distribution of heat generation with the device

- temperatures in the ceiling void

\section{Calibration approach}

An initial guess at the device cooling air flow rate, the room extract rate and lighting use was applied to the model and the predictions showed that the device exhaust was $\sim 50-70^{\circ} \mathrm{C}$.

The flow rate and fan on-temperature were adjusted to lower the exhaust temperature. The room exhaust was also adjusted to 6 litres/s to approximate the fresh-air control in the adjacent offices.

It was noticed that the heat from newly printed paper had not been accounted for so two of the surfaces in the sorter were converted to steel plates to approximate the hot paper temperatures. During test runs the internal mass temperatures were seen to be decoupled from the heat injections and with the assumption that air flows within the device would not be well represented by the default treatment, alternative heat transfer coefficients were imposed. In all there were five iterations required to identify and adjust the response of the device toward that of the monitored data. In Figure 15 the turquoise line is .

Given the observation of mild discomfort near the photocopy machine MRT sensors at the operator's position in the room and near the doorway were added and view factors were also computed so that position specific comfort calculations could be carried out.

For comparison, the photocopy room should also be simulated with an abstract scheduled casual gain representation of the photocopy machine as well as tested in the context of an open plan office with adjacent desks.

This study indicates the types of performance information which can be derived from explicit representations of office equipment. A more heavily loaded photocopy machine would, or course, require an alternative power demand profile but otherwise a standard pre-defined entity and sets of electrical demand profiles would be broadly applicable. Machines located in open plan offices would expected to create a local heat island which would disperse into adjacent work areas. Here increasing the resolution of the solution via a CFD domain might be considered.

\section{Heat exchangers}

Mechanical ventilation with heat recovery (MVHR) devices which combine fans and heat exchangers in a compact unit are increasingly ubiquitous (Figure 16). Their efficiency is calculated in various ways and one is shown below. Many simulation tools offer system components, possibly linked to a flow network to provide control (although staff in a hurry often resort to re-setting infiltration rates). ESP-r offers a component based approach using systems components, but users often then simplify the air flow regime. For buildings with hybrid designs where natural air flows and mechanical flows mix a zonal approach may be a better fit. This case study looks at representing the air as it flows through a series of thermal zones with surfaces forming the heat exchanger with suitably set surface heat transfer coefficients. This approximates the heat transfer paths one might find in a MVHR. The model approximates the MVHR case and ancillary components (Figure 17). This was compared with an ESP-r systems component approach designed with the same characteristics.

In the model tempered air is supplied to the left room via the explicit heat exchanger and the left_alt room via system components. In each case the air flows to the right or right_alt zone and passes out through either the explicit heat exchanger or the relevant system components. 
The inter-zone surfaces in the heat exchanger zones approximate the heat exchange area. Heat transfer coefficients are set for the typical velocity in the MVHR. In this case, passing air through three zones in each path captured most of the temperature transition observed in a counter-flow heat exchanger yielding $\sim 75-80 \%$ efficiency. The alternative plant network approach includes ducts (with equivalent flow components), a fan and three heat exchanger components (to represent the counter-flow and transition zones of the MVHR). The rooms have the same diversity of use and are controlled to the same temperatures.
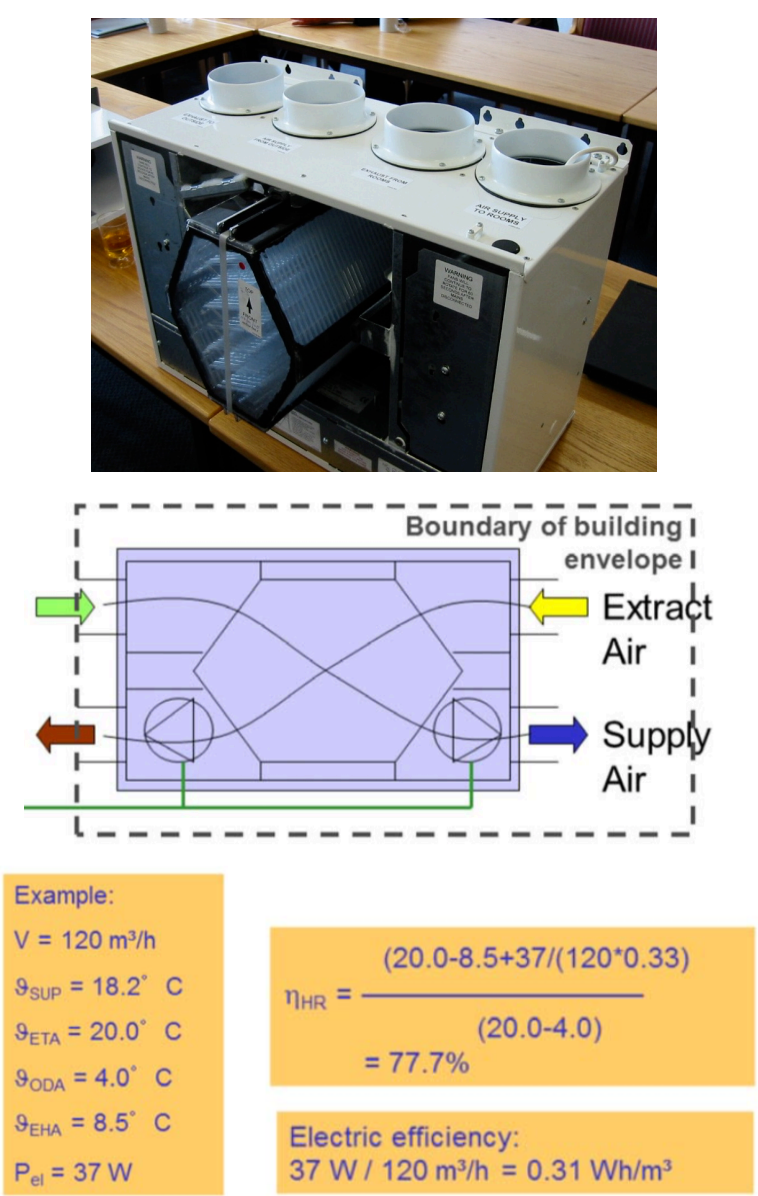

Figure 16: Mechanical ventilation heat recovery unit.

The temperatures at various points in the heat exchanger are shown in Figure 18 and are roughly as expected. As all other zones attributes are the same, the equivalence of the two representations of tempering the incoming air should show up in the pattern of heating required. The heating demands (Figure 19) follow a similar pattern but there is a shift in magnitude between the two methods which is not yet explained.

In many ways it is early days for a zonal approach as there are many attributes of MVHR units which have been assumed. Certainly this approach does not track real-world issues such as condensation droplets in the heat exchanger or vapour permeable membranes where latent transfers also occur. Further calibration is warranted.

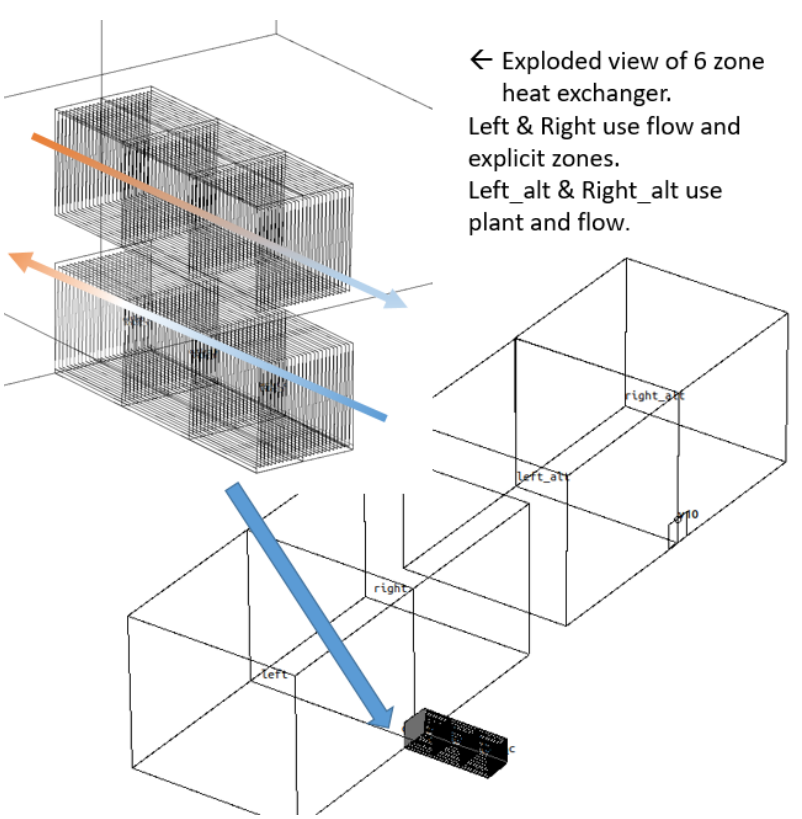

Figure 17: Exploded view of HE and used with zones.

What is not included in this representation are heat gains from the fans in MVHR. This would require additional geometric resolution. There are also no filters or a facility for a 'summer bypass'. The space taken up by the virtual MVHR is not quite the same as the physical devices so there is further work needed if this approach was to be taken forward.

A three-zones-along-the-heat exchanger yields temperature patterns consistent with $\sim 80$ efficiency. Additional zones would be needed to support units of extreme efficienty. What is missing are experimental data to better characterise the transition temperatures at the air entry points.

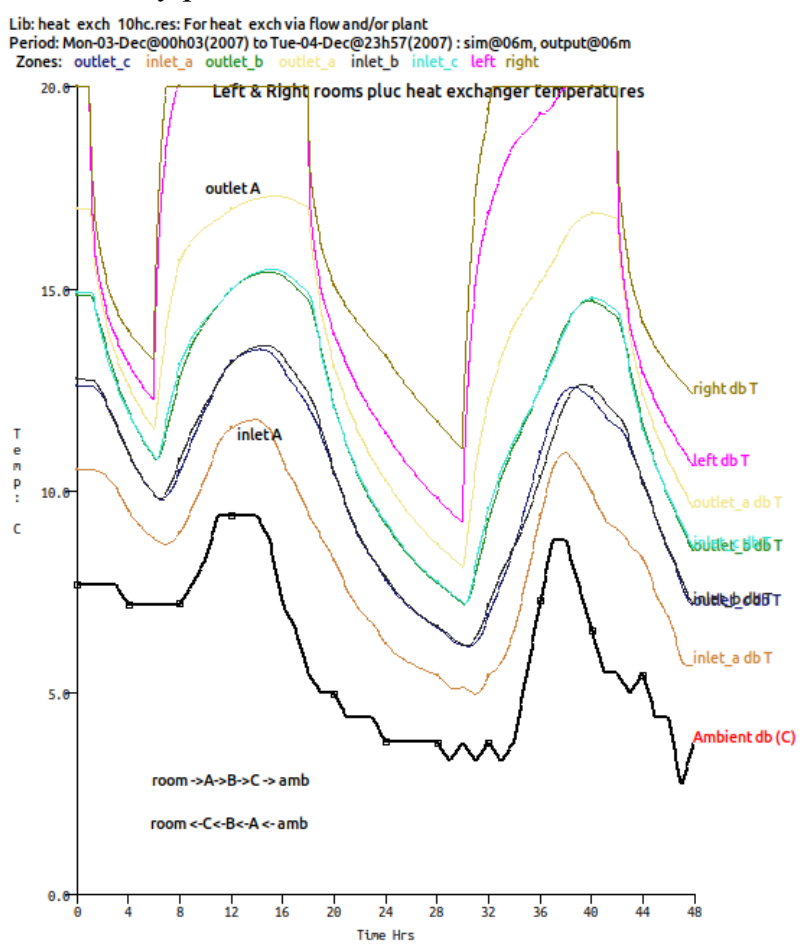


Figure 18: Temperatures at various points in heat exchanger.

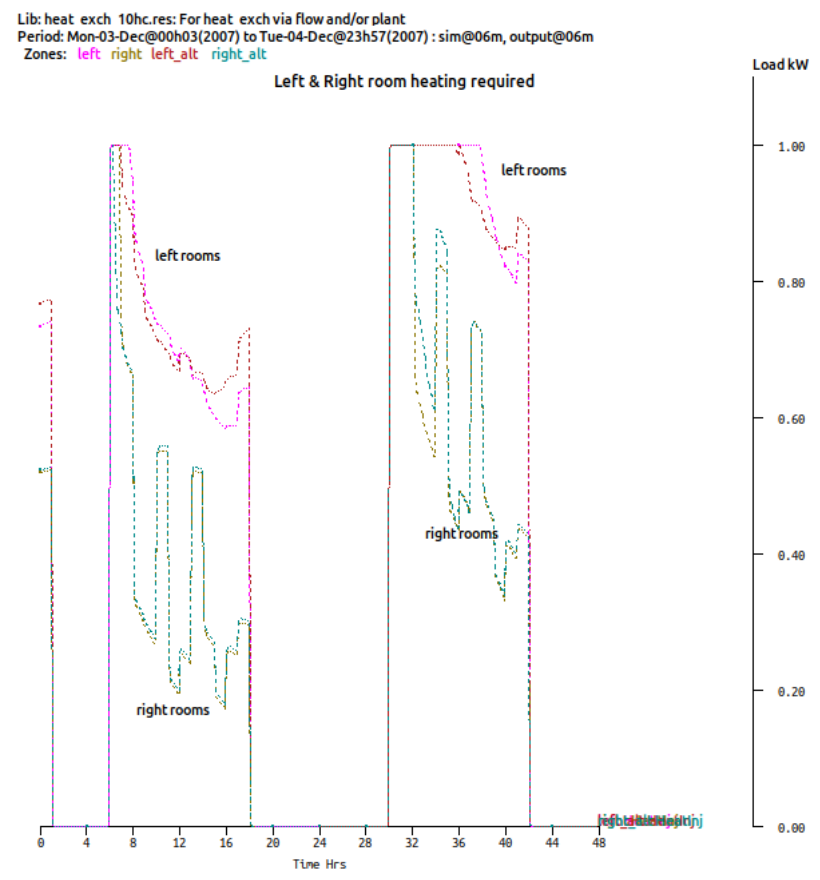

Figure 19: Heating demands for both approaches.

\section{Conclusions}

Several case studies have been used to explore zonal approaches to representing the performance of sub-zone scale artefacts within a whole-building simulation suite. We have observed:

- Zonal representations involve a step-change in the number of surfaces and zones in a model. This increases run-times as well as the size of simulation results files.

- For entities, such as the photocopy machine, which have been added to ESP-r's library of pre-defined entities, inclusion in the model is streamlined but some attribution involves mass flow components and patterns of electrical demand which the user must still manage manually. This also applies to radiators and other system entities that involve multiple solution domains. Work is needed to extend the data model to include such attributes.

- There is also a step change in performance information e.g. energy balances at each of the zones and surfaces as well as their temperatures an all of the flux paths normally associated with surface entities. As a simulation community we have not yet evolved working practies to take into account such information in evaluating design decisions.

- Where mass flows and heat transfer between surfaces and air are involved we have found that these are best captured if we move to shorter simulation timesteps say 5 minutes rather than 15 minutes. If control is to be imposed, a 1-2 minute timestep is sometimes required.

- Because we are still in the early stages of learning, there are few rules as to the specific level of detail needed to approximate these artefacts. Although we can use a caliper to measure the dimensions within a thermostat further research is needed to establish how much room air actually mixes within such devices.

- Some topics are still very much work in progress. Thermostic radiator valves are crudly represented and would require quite a bit of work. Losses in pipe-work would require that heat flows from mass flow components are tracked - probably a $\mathrm{PhD}$ in that topic.

- Calibration is an iterative process. It helps to have physical devices to observe and measure but there are any number of uncertainties in the form and composition and it will take time to explore sensitivities.

- Some of these approaches do not scale well. Including a dozen MVHR units in an office building would likely require considerable passion.

- One of the attractions of explicit representations is that is supports detailed comfort assessments and high resolution heat exchanges between objects and their surroundings. Most system component positions and exchanges with their surroundings are rather abstract. From the current perspective we might focus on methods to anchor system componets in space and enhance their interaction with their surroundings.

\section{Acknowledgement}

The author would like to thank his colleagues in (xx) who assisted in the monitoring of some of the devices tested as well as assisting with setting up of the case study models.

\section{References}

Clarke J.A. 2001. Energy Simulation in Building Design ( $2^{\text {nd }}$ edition) Butterworth Heinemann, Oxford.

Cockroft J. (2017-2018) A series of interviews in Glasgow Scotland.

Hand J.W. (2016). Performance implication of fully participating furniture and fittings in simulation models. Proceedings of BSO16 Building Simulation \& Optimization 2016. Newcastle (UK).

Hand J.W. (2018). Strategies for Deploying Virtual Representations of the Built Environment. ESRU, University of Strathclyde, Glasgow (UK).

Larson G. Shakespeare R. 1998. Rendering with Radiance. Morgan Kaufmann, San Francisco, California.

U.S Department of Energy (2017). EnergyPlus Version 8.8.0 Documentation Input Output Reference. Technical Information Center, United States Department of Energy, NTIS, Springfield Virginia (USA). 
